ARTICLE

\title{
Single-cell measurement of plasmid copy number and promoter activity
}

Bin Shao', Jayan Rammohan (D) ${ }^{2}$, Daniel A. Anderson (10 ${ }^{1}$, Nina Alperovich², David Ross (iD ${ }^{2}$ \& Christopher A. Voigt (1) ${ }^{1 凶}$

Accurate measurements of promoter activities are crucial for predictably building genetic systems. Here we report a method to simultaneously count plasmid DNA, RNA transcripts, and protein expression in single living bacteria. From these data, the activity of a promoter in units of RNAP/s can be inferred. This work facilitates the reporting of promoters in absolute units, the variability in their activity across a population, and their quantitative toll on cellular resources, all of which provide critical insights for cellular engineering. 
A ccurate measurements of promoter activities are crucial for understanding the biophysics of transcription and enable the predictive construction of genetic systems ${ }^{1}$. The strength of a promoter can be described as the flux of transcribing RNA polymerases (RNAPs) exiting a promoter $(\mathrm{RNAP} / \mathrm{s})^{1}$. In essence, this is the time- and population-averaged output of the biophysical complexity of an individual promoter, which involves abortive short transcripts, pausing, and bursts of activity $^{2-4}$.

In the design of genetic circuits, promoters carry the signal between sensors and gates ${ }^{5-7}$. In metabolic engineering, enzyme levels can be balanced by selecting promoters of different strength $^{8}$. Increasingly, these tasks are being performed by computer aided design (CAD), the precision of which is limited by part measurement accuracy 7,8 . The strength of promoters in absolute units is rarely known and is more often measured indirectly with a reporter gene and provided in "arbitrary units"9. It has been proposed to define a constitutive promoter $\left(\mathrm{P}_{\mathrm{J} 23101}\right)$ carried on a $\mathrm{p} 15 \mathrm{~A}$ plasmid as a reference, to which a promoter-ofinterest is compared and the strength reported in relative promoter units (RPUs) ${ }^{10}$. The conversion of $1 \mathrm{RPU}$ to RNAP/s has been estimated ${ }^{7,10}$.

Single-molecule methods to visualize transcribing RNAPs exiting a promoter are either performed in vitro or are technically challenging ${ }^{11-14}$. Across a population, the same promoter may have different activities in each cell because of extrinsic noise; in other words, differences in cellular resources such as RNAPs and plasmids ${ }^{15-17}$. Promoter activity can be inferred from mRNA transcripts, but methods such as single molecule fluorescence in situ hybridization ( $\mathrm{smFISH}$ ) require fixing the cells and singlecell RNA-seq loses the required resolution ${ }^{4,11,18-21}$.

A problem with using bulk measurements to calculate promoter activity is that each cell has a different number of copies of the promoter because of differences in the copy number of the plasmid or genome on which it is carried. Plasmid copy number is dictated by its replication origin and can change depending on the genetics of the host strain ${ }^{22}$ and growth conditions ${ }^{23}$ and the copy number of the genome varies depending on the distance to the origin and growth rate 24,25 . The average plasmid copy number has been estimated with bulk DNA measurements, but no method has been developed to count the plasmid copy number in single living cells. Plasmids can be visualized using DNA FISH and super-resolution microscopy or by fusing DNAbinding proteins to fluorescent reporters, but they have not been calibrated to provide absolute units ${ }^{26-33}$. PCR-based methods suffer from accuracy and is difficult to implement for single cell measurements 34,35 .

In this work, we develop a method to use fluorescent reporters fused to binding proteins that label the plasmid and RNA transcripts so that they, along with protein expression, can be measured simultaneously in individual cells. Inspired by earlier work to use DNA-binding proteins to detect plasmids in vivo ${ }^{28-33}$, we use PhlF fused to red fluorescent protein (RFP) to count plasmids. In the same cell, we use the PP7 RNA-binding protein fused to cyan fluorescent protein (CFP), selected because it requires the fewest repeated operators and has minimal impact on mRNA degradation ${ }^{36-46}$. These data enable us to calculate the activity of promoters in absolute units in individual living cells. Further, we are also able to simultaneously detect protein expression using yellow fluorescent protein (YFP).

\section{Results}

Quantification of the plasmid copy number in single cells. Plasmids are detected in individual cells using fluorescence microscopy (Fig. 1a, b). The backbone is modified to insert a region with 14 operator repeats that bind the $\mathrm{PhlF}$ repressor ${ }^{47}$, flanked by strong terminators (Fig. 1a and Supplementary Fig. 2). Note that this is much smaller than previous work; for example, the use of 240 tet $O$ sites to bind $\mathrm{TetR}^{26,28}$. This plasmid also contains the green fluorescent protein gene $(g f p)$ under the control of a constitutive promoter, allowing for the simultaneous measurement of plasmid copy number and protein expression. From a second plasmid ( $p$ SC101 origin), a PhlF-RFP fusion protein is expressed under the control of an aTc-inducible promoter. The plasmids are transformed into $E$. coli $\mathrm{NEB} 10 \beta$ and grown at $37^{\circ} \mathrm{C}$ in $\mathrm{M} 9$ media until reaching exponential phase in the presence of aTc, ampicillin and kanamycin (Methods). An aliquot of cells is taken, placed on a cover slip with an agar slab and imaged using an inverted fluorescence microscope.

The copy number was determined for different origins of replication (ori) (pSC101, p15A, pColE1, and pUC). (To measure pSC101, the phlF-rfp expression cassette is on the same plasmid, Supplementary Fig. 2). As the copy number increases, punctate red spots become brighter and more abundant (Fig. 1b, Supplementary Fig. 1 and Supplementary Fig. 2) (Methods). When the spot intensity data are plotted for the lowest copy plasmid (pSC101) as a histogram, equidistant peaks are apparent (Fig. 1c). The mean distances between the first four peaks were used to determine the spot intensity due to one plasmid. Then, the mean plasmid copy number per cell is calculated for each backbone: 4 (pSC101), 9 (p15A), 18 (pColE1), and 61 (pUC) (Fig. 1d and Supplementary Fig. 2). These means are consistent with previous bulk measurements ${ }^{34,48}$. The numbers of plasmids per spot were also consistent with previous measurements of plasmid clusters within cells; for example, for pColE1, each plasmid spot contains 11 plasmids on average, which is similar to the cluster size $(\sim 10)$ revealed by quantitative localization microscopy ${ }^{18}$. Plasmid clustering could be explained by multimerization, in which plasmids form high order oligomers as a result of recombination between individual plasmid molecules, or the sharing of replication machinery ${ }^{49}$.

The distribution of plasmid copy numbers across a population of cells is shown in Fig. 1e. The distributions are wide, with standard deviations on the order of the mean copy numbers (4, 11, 15 and 60 for pSC101, p15A, pColE1, and pUC). The extreme ends of the distributions were notable, with a large fraction of cells lacking plasmid entirely $(5 \%, 3 \%, 1 \%$ and $1 \%)$ and many cells containing 4 -fold or more of the mean copy number. We found these distributions were consistent between day-to-day measurements, antibiotic choice, and protein expression cassettes (Supplementary Fig. 3). Even the incorporation of a toxin-antitoxin system (hok/sok), which has been used to minimize plasmid loss ${ }^{50}$, results in the same distribution.

We developed a mathematical model to determine if these distributions would emerge from simple rules for plasmid replication and cell division (Fig. 1f) (Methods and Supplementary Note 1). Plasmids are first distributed across a population for a desired average copy number $N_{0}$. Then, iterations of growth and division are performed: cells are selected randomly, plasmids are distributed following a modified binomial distribution (partitioning coefficient, $a)^{51,52}$, and they replicate with feedback ${ }^{51,53}$ (sensitivity, $K$ ) until $N_{O}$ is reobtained. The shape of the distribution is determined by the tightness of plasmid copy number control and partitioning, with more even partitioning leading to less plasmid loss and tighter copy number control resulting in lower variance of the distribution. With two fit parameters ( $a$ and $K$ ), this converges on the observed plasmid distributions from disparate starting distributions (Supplementary Fig. 4). The prediction errors could be due to additional sources of randomness in plasmid segregation. The parameter values 


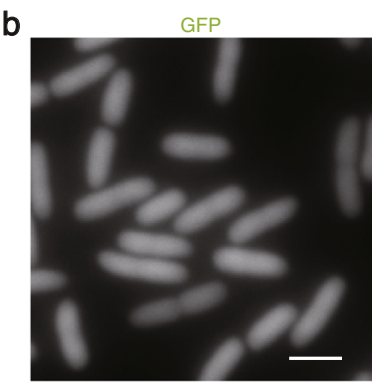

e

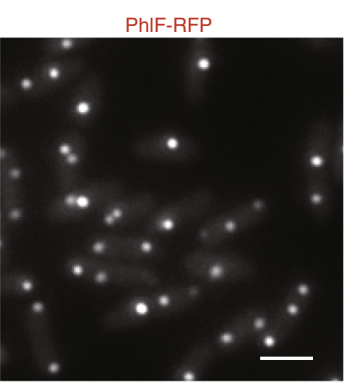

f

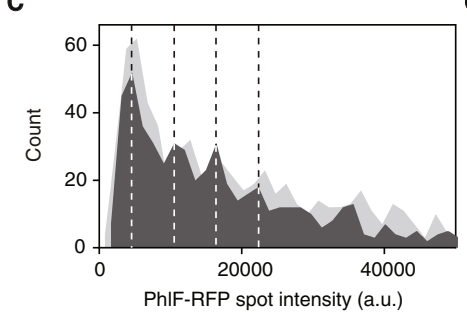

d

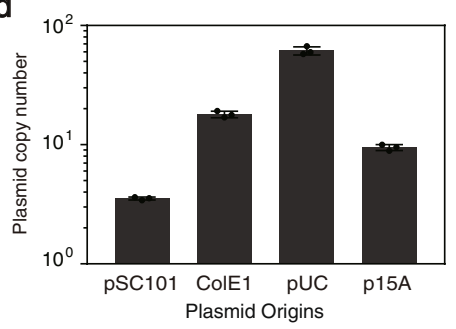

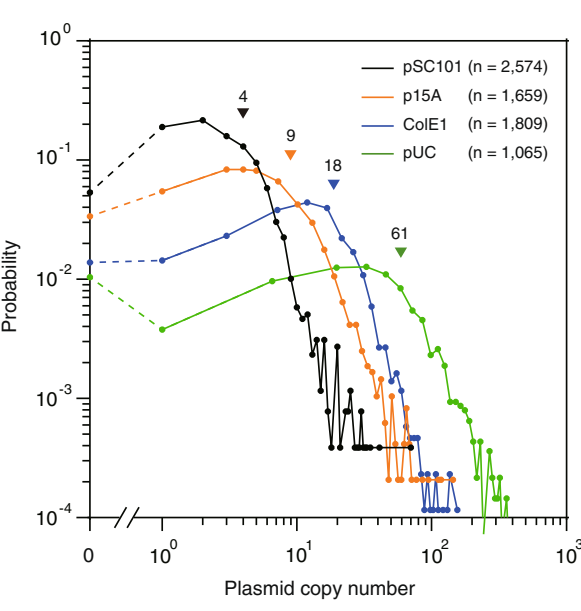

remove half of cells

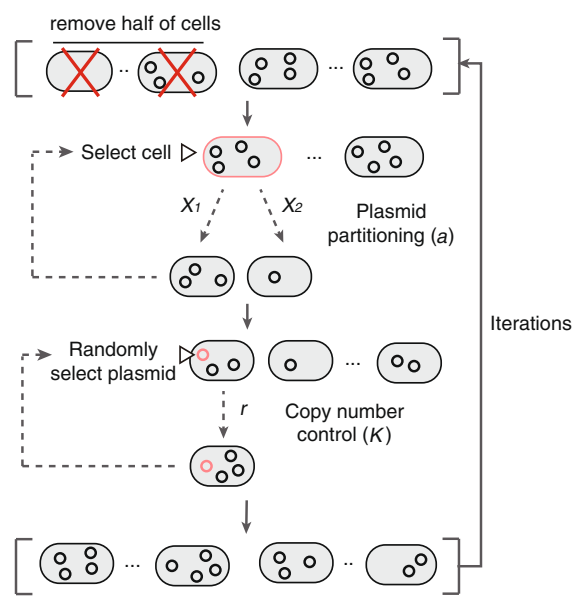

g

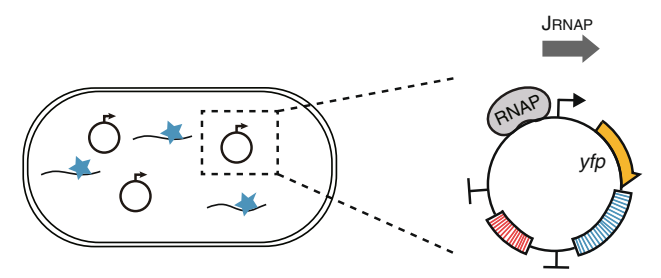

h

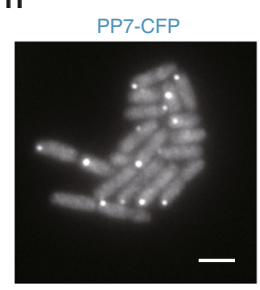

PP7-FISH

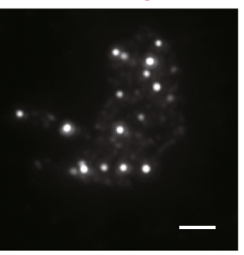

Merge

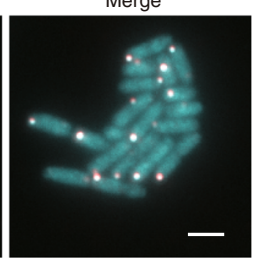

i

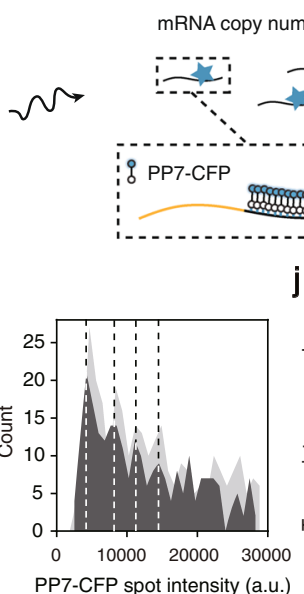

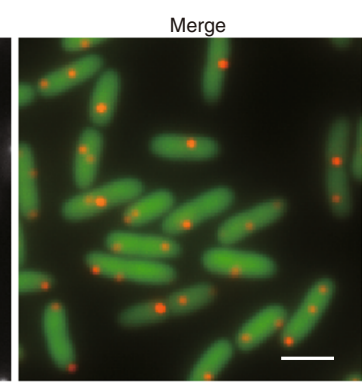

(

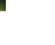


Fig. 1 Measurement of plasmid copy number and transcript number. a Schematic of the plasmid copy number calibration construct. PhIF-RFP is expressed from a second plasmid (pSB235-237). "Ori" indicates where the plasmid origin is changed in the plasmid being measured. Plasmid maps and part sequences are provided in Supplementary Fig. S18 and Table S3. b Images are shown for the measurement of ColE1 plasmids (pSB220) in the cell. GFP is the expression of the reporter gene $(525 / 50 \mathrm{~nm})$ and PhIF-RFP shows the binding of the reporter to the plasmids, where brighter spots indicate more plasmids in a cluster $(645 / 75 \mathrm{~nm})$. Scale bar, $2 \mu \mathrm{m}$. c The histogram of PhIF-RFP spot intensity from the low copy pSC101 backbone is shown. The dashed lines show the peaks, the distance between them is used to calculate the spot intensity of individual plasmids. The light and dark gray histograms show the distributions from replicates performed on different days. $\mathbf{d}$ Measured mean copy number for different plasmid backbones. The means are calculated from three replicates performed on different days and the error bars are the standard deviations of these experiments. e Copy number distributions across a population of cells are shown. The triangles indicate the means of the population. The distributions are made from a combination of 3 replicates performed on different days (cells numbers from each replicate is 858 for pSC101, 553 for p15A, 603 for ColE1 and 355 for pUC, p values from the two-sample Kolmogorov-Smirnov test for pooling the replicates are 0.97/0.58/0.10 for pSC101, 0.89/0.97/0.67 for p15A, 0.76/0.22/0.72 for ColE1, 0.61/0.98/0.33 for $\mathrm{pUC}$ ). $\mathbf{f}$ The model for simulating the convergence onto a plasmid copy number distribution (Supplementary Note 1). The two daughter cells get $x_{1}$ and $x_{2}$ plasmids. $a$ is the partitioning coefficient and $K$ is the sensitivity for plasmid replication control. $\mathbf{g}$ Schematic showing the detection of mRNA in single cells. PP7 binding sites (blue) are shown on the pSB223 plasmid and mRNA. This plasmid is co-transformed with pSB233, which contains the genes for PP7-CFP and PhIF-RFP expression (not shown). h Overlap of the FISH signal targeting PP7 binding sites with the PP7-CFP signal (Methods). Scale bar, $2 \mu \mathrm{m}$. $\mathbf{i}$ The histogram of PP7-CFP spot intensity from a plasmid expressing small amounts of mRNA is shown $\left(P_{\text {tad }} / 20 \mu M\right.$ IPTG/pSB208). The dashed lines show the peaks, the distance between them is used to calculate the spot intensity of individual mRNAs. The light and dark gray histograms show the distributions from replicates performed on different days. $\mathbf{j}$ Quantification of mRNA copy number for a library of promoters on p15A plasmid backbone. Plasmid maps and part sequences are provided in Supplementary Fig. S18 and Table S3. k Dynamics of transcript copy number from the constitutive promoter $P_{J 23101}$ after rifampicin addition. The data was fitted to a single exponential decay. The resulting degradation half-life $(\mu)$ is $6.8 \pm 0.3$ min. The impact of rifampicin addition on YFP expression and plasmid copy number is shown in Supplementary Fig. S10. For parts b and h, microscope experiments were repeated three times with similar results. For parts $j$ and $k$, the means were calculated from the population means from three replicates performed on different days and the error bars represent the standard deviation of these means. Source data are provided as a Source Data file.

promoters $^{55,56}$. No crosstalk is observed between the DNA- and RNA-binding fusion proteins (Supplementary Fig. 7); therefore, they can both be used to simultaneously measure DNA and mRNA copy numbers in single cells. In addition, this technique can be used to label other RNAs; for example, the small guide RNA (sgRNA) that bind to dCas9 (Supplementary Fig. 8).

Our calculation of the fractions of cells containing no mRNAs or plasmids could be due to less-than-perfect detection efficiency. We sought to determine whether this could be due to cells that have the target molecule, but did not get labeled due to a fluctuation in the labeling protein. Cells for which we found no mRNA transcripts do not have a lower total PP7-CFP expression (Supplementary Fig. 6e). Changing the PP7-CFP expression level does not change the fraction without mRNA: $5 \%$ when high and 6\% when low (Supplementary Fig. 6f). Further, these numbers are consistent with previous results from smFISH experiments $(5-8 \%)^{11,55}$. The FISH intensity and PP7CFP spot intensity are strongly correlated $\left(R^{2}=0.95\right.$ Supplementary Fig. 6). Similarly, the number of plasmids per cell and the fraction of cells without plasmid is not impacted by the total PhlF-RFP expression (Supplementary Fig. 2j, k). Cells without detectable PhlF-RFP spots also have very low GFP expression, supporting that it is the result of plasmid loss rather than detection error (Supplementary Fig. 2).

Single cell measurement of promoter activity. Inferring the activity of a promoter in units of RNAP flux requires promoter copy number, mRNA copy number and degradation rate. The productive RNAP flux is the same as the generation rate for complete mRNAs. The flux in units of RNAP per second per DNA (RNAP/s-DNA) is calculated as $\bar{J}_{R N A P}=\tau(m / N)$, where $\tau$ is the mRNA degradation rate, $m$ is the mRNA copy number and $N$ is the plasmid copy number. The half-life of mRNA $(\mu=\ln (2) / \tau)$ in $E$. coli is typically in the range of several minutes ${ }^{22,23}$. Using a rifampicin assay (Methods), we measured a mean half-life of 6.8 $\pm 0.3 \mathrm{~min}$ for transcripts from $\mathrm{P}_{\mathrm{J} 23101}$ driving $y f p$ and the PP7 repeat in the presence of PP7-CFP (Fig. 1k, Supplementary Fig. 9, Supplementary Fig. 10). Note that using a population-averaged mRNA degradation rate is an approximation because of extrinsic noise in degradation machinery and variability in mRNA partitioning ${ }^{41,57}$.

From these data, we calculated $\bar{J}_{R N A P}$ from the reference promoter $\mathrm{P}_{\mathrm{J} 23101}$ in individual cells (Fig. 2a and Supplementary Fig. 11). The fluorescence distribution from this promoter (YFP) and cell growth rate are not affected by the addition of the binding operators nor co-transforming with the plasmid containing the fusion proteins. The promoter activity is measured in each cell by simultaneously measuring the mRNA and plasmid copy numbers (Fig. 2b), while assuming the degradation rate is constant. The distribution of promoter activities for a population of cells in the same experiment is shown in Fig. 2c (only for those cells in which DNA is detected). The population mean is $<\bar{J}_{R N A P}>=\tau<m / N>=0.019 \mathrm{RNAP} / \mathrm{s}-\mathrm{DNA}$, similar to the values estimated in the literature ${ }^{7,10}$. However, the distribution is broad: the standard deviation across a cell population from a single experiment is $0.028 \mathrm{RNAP} / \mathrm{s}-\mathrm{DNA}$ whereas the standard deviation of the population means from measurements from different days is $0.002 \mathrm{RNAP} / \mathrm{s}$-DNA (Fig. 2c). The population is bimodal because no mRNA is detected in $5 \%$ of the cells in which plasmids can be detected. This bimodality is not observed in protein expression (YFP) either by microscopy or cytometry (Fig. 2d). This could be explained by the long half-lives of reporter proteins, which averages out the fluctuations in mRNA numbers. Our results demonstrate that the measurement of a highly-expressed fluorescent reporter does not capture the underlying population behavior.

We took time lapse movies of bacteria growing on an agar pad (Supplementary Fig. 12). For each cell, the dynamics of RFP, GFP, and YFP were measured, setting cell division to $t=0$. The plasmid and mRNA copy number decrease after cell division and increase during the cell cycle, as expected. The promoter activity is initially 3 -fold higher, before converging to the average distribution after $5 \mathrm{~min}$. This observation may be due to divergence from the pseudo-steady-state approximation, where the mRNA and protein levels need to adjust to the new DNA concentration after division.

The promoter activity reports the RNAP flux from a single copy of the promoter. Each plasmid carries one promoter and the sum of their activities in a cell is referred to as the "total promoter activity". Using these definitions, we explored how the promoter 
a

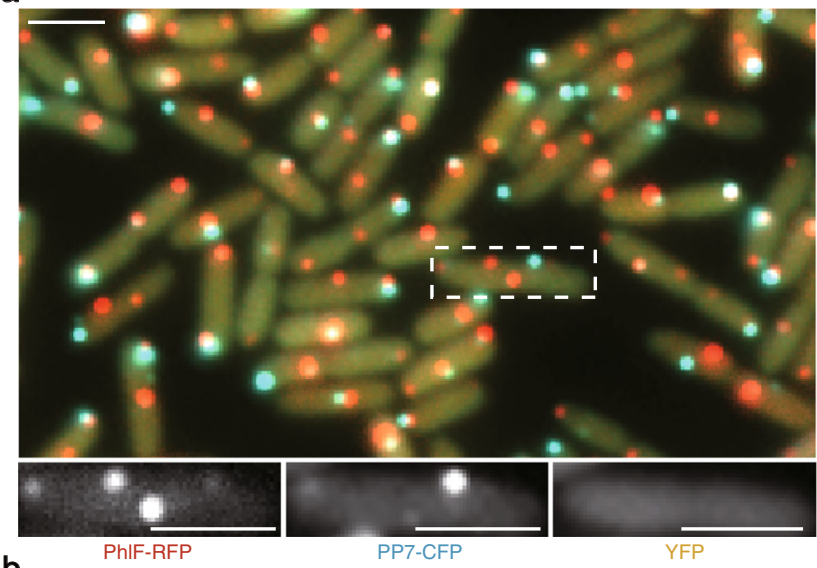

b

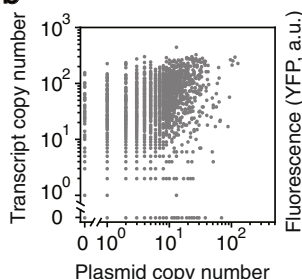

C

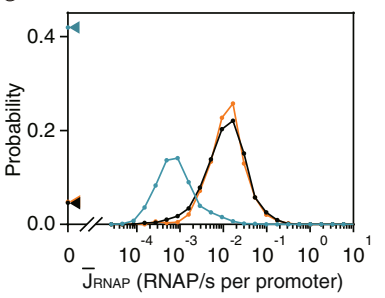

e

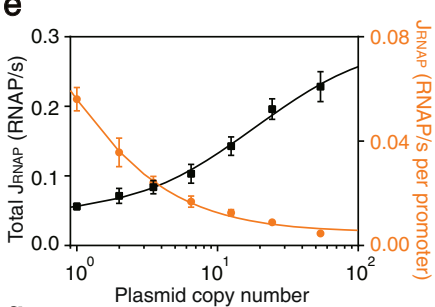

g

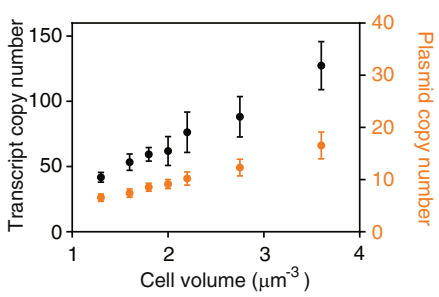

f

d

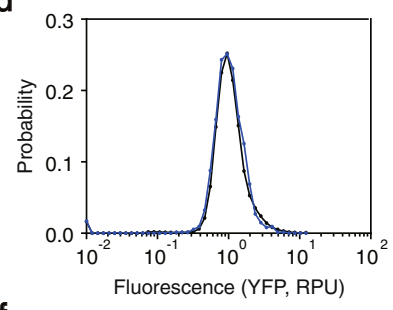

f

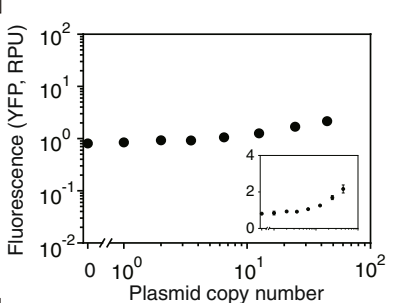

$\mathrm{h}$

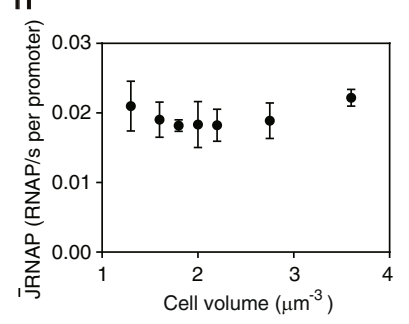

activity changes as a function of the number of promoters carried in a cell. As expected, the total activity is higher when a cell contains more plasmids (Fig. 2e and Supplementary Fig. 13). For cells with a fixed number of plasmids, the transcript distribution is wider than a Poisson distribution, which could be explained by a two state promoter model ${ }^{58}$ (Supplementary Fig. 13). The total promoter activity does not grow linearly with copy number. Rather, the activities of individual promoters decline, which could be due to a limit in the number of available RNAPs in the cell $^{59,60}$. Therefore, a 100-fold change in plasmid copy number is reduced to a 4 -fold change in total promoter activity and further reduced to 2 -fold in terms of YFP expression (Fig. 2f). This has a profound impact on the use of promoters for genetic circuits or
Fig. 2 Inference of promoter activity in individual cells. a Sample images showing simultaneous quantification of DNA (RFP), RNA copy number (CFP) and protein production (YFP) for $\mathrm{P}_{\mathrm{J} 23101}$ carried on pSB223 (Supplementary Fig. S1). PP7-CFP and PhIF-RFP are induced with $6 \mathrm{ng} / \mathrm{ml}$ aTc (pSB233) (Supplementary Fig. S11). Scale bar, $2 \mu \mathrm{m}$. Contrast/ brightness for different color channels are changed individually to adjust the color balance (Supplementary Fig. S14). Microscope experiments were repeated three times with similar results. b Each point shows a measurement from an individual cell (data obtained from 5 images taken from independent experiments, $n=2178$ cells). From left-to-right, the $\mathrm{R}^{2}$ to a linear regression model in log scale are: DNA vs mRNA (0.13), DNA vs protein (0.20) and mRNA vs protein (0.14). c The calculated promoter activites of $P_{J_{23101}}$ in single cells. The black and blue distributions are when cells are grown in M9 media to exponential phase and stationary phase, respectively (Methods, $n=2178$ cells and $n=1863$ cells). The orange distribution ( $n=1344$ cells) is when cells are grown in $2 \times Y$ T media. The distributions are made from a combination of three replicates performed on different days. Dots are experimental data with lines to guide the eye. Only those cells for which plasmid can be detected are included in the distributions. The triangles indicate the percent of cells where no promoter activity is detected, but where plasmid can be observed. d Single-cell YFP fluoresence from $\mathrm{P}_{\mathrm{J} 23101}$ measured by quantitative microscope (black dots) and flow cytometry (blue dots). The medians are scaled so that they are at $\mathrm{RPU}=1$. The distributions are made from a combination of 3 replicates performed on different days. e The total RNAP flux from all promoter copies (black) and the per promoter flux (orange) are shown as a function of the plasmid copy number in individual cells. $\mathbf{f}$ The YFP expression is shown as a function of the plasmid copy number in individual cells (inset, YFP expression in linear scale). $\mathbf{g}$ The transcript copy number (black) and plasmid copy number (orange) are shown as a function of the cell volume. $\mathbf{h}$ The RNAP flux per promoter is shown as a function of the cell volume. For part $\mathbf{e}$ and $\mathbf{f}$, the single cell data is binned by plasmid copy number. The lines show the best fit to a Hill equation. For part $g$ and $h$, the single cell data is binned by cell volume. For part $\mathbf{e}, \mathbf{f}, \mathbf{g}$ and $\mathbf{h}$, data are presented as mean values and the error bars represent the standard deviation of these measurements from three experiments performed on different days. Source data are provided as a Source Data file.

enzyme balancing as large cell-to-cell fluctuations are buffered that would otherwise cause errors. In addition, it speaks to the futility of using high copy number plasmids to increase heterologous protein expression, where there is a diminishing return as well as a disproportionate drain on cellular resources to carry the additional plasmid copies. We also found the per promoter RNAP flux is largely constant for cells of different volumes (Fig. $2 \mathrm{~h}$ ), even when the transcript copy number is highly correlated with the cell volume (Fig. $2 \mathrm{~g}$ ).

Media and growth phase can impact the plasmid copy number. The plasmid copy number is higher (increases to 13) for cells growing in rich media $(2 \times Y T)$ (Supplementary Fig. 14). There's also an increase in the transcript copy number and the promoter activity distribution is similar to the cells growing in M9 media (Fig. 2c). In stationary phase, the promoter activity decreases to $<\bar{J}_{R N A P}>=0.001 \mathrm{RNAP} / \mathrm{s}$-DNA and the fraction showing no activity increases to $41 \%$, consistent with $\sigma 70$ being unavailable (plasmid copy number also increases to 17) (Fig. 2c and Supplementary Fig. 14). As the result of growth arrest, the YFP fluorescence is higher in stationary phase even when transcriptional activity is low (Supplementary Fig. 14).

The system was then used to measure plasmid copy number and the activity of the $\mathrm{P}_{\mathrm{J} 23101}$ in other strains (Fig. 3, Supplementary Fig. 15). First, we tested E. coli MG1655, which is closer to wild-type than E. coli NEB10-beta. The copy number distributions for the 


\section{E. coli MG1655}
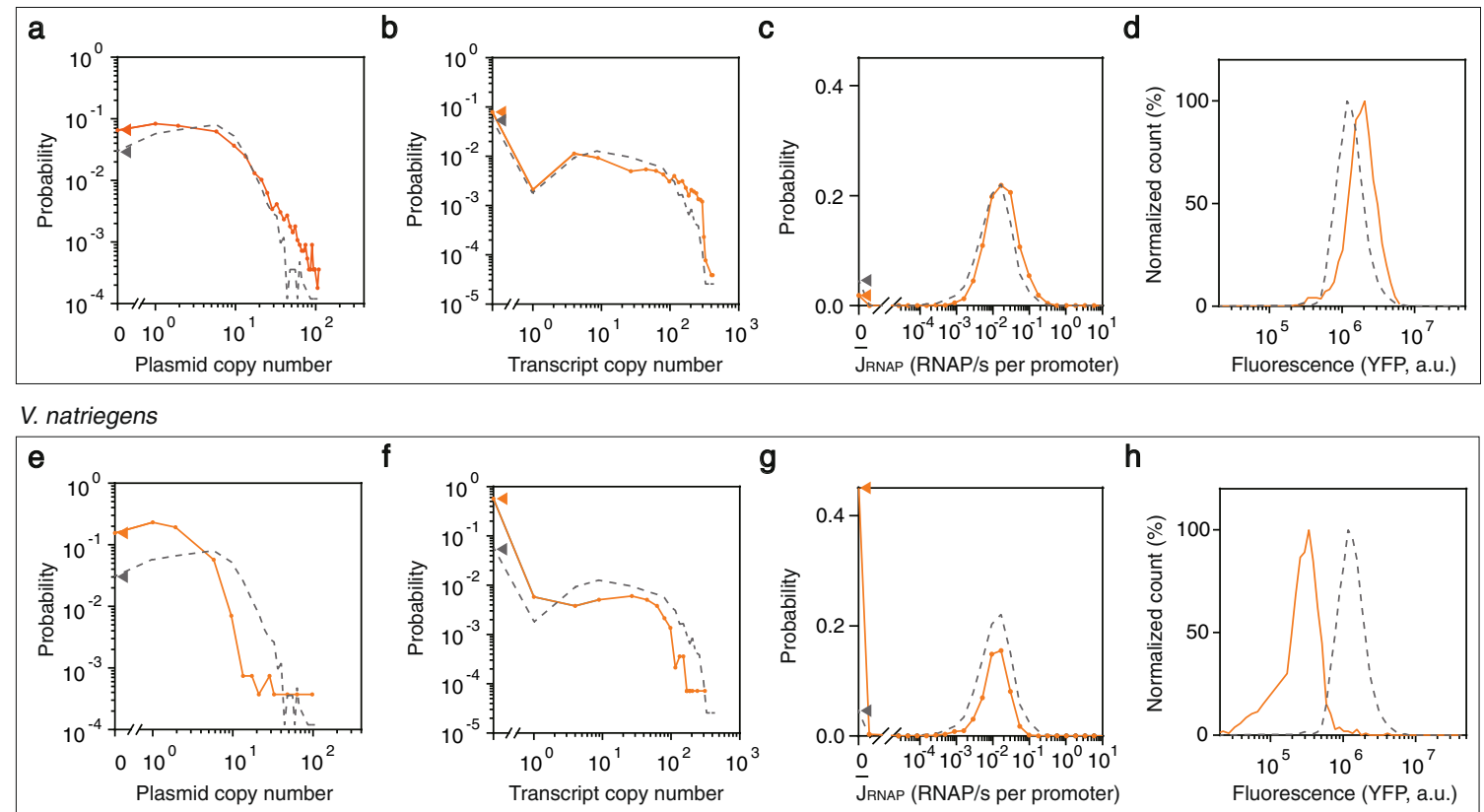

Fig. 3 Calculation of plasmid copy number and promoter activity across different strains. The dashed lines in all graphs are the distributions for $E$. coli NEB 10-beta. a Plasmid copy number $(n=1449, p$-values from the two-sample Kolmogorov-Smirnov test for pooling the replicates are 0.26/0.92/0.43). b mRNA copy number $(p=0.38 / 0.99 / 0.69)$. c Promoter activites of $\mathrm{P}_{\mathrm{J} 23101}$. d The YFP fluorescence distribution. e Plasmid distribution for $V$. natriegens $(n=783, p=0.55 / 0.05 / 0.77)$. $\mathbf{f}$ mRNA copy number $(p=0.25 / 0.35 / 0.62)$. $\mathbf{g}$ Promoter activites of $\mathrm{P}_{\mathrm{J} 23101} \mathbf{h}$ The YFP fluorescence distribution. All the distributions are made from a combination of three replicates performed on different days. For part $\mathbf{a}, \mathbf{b}, \mathbf{c}, \mathbf{e}, \mathbf{f}$ and $\mathbf{g}$, dots are experimental data with lines to guide the eye. For part $\mathbf{a}, \mathbf{b}, \mathbf{e}$ and $\mathbf{f}$, the triangles indicate the percent of cells where no plasmid or transcript is detected. For part $\mathbf{c}$ and $\mathbf{g}$, the triangles indicate the percent of cells where no promoter activity is detected, but where plasmid can be observed. Source data are provided as a Source Data file.

p15a plasmid are very similar (average of $13.2 \pm 1.8$ ), although plasmid loss is $\sim 2$-fold higher, possibly due to the presence of DNA modifying enzymes. The average promoter activity is slightly higher $\left\langle\bar{J}_{R N A P}\right\rangle=0.029 \mathrm{RNAP} / \mathrm{s}$ - DNA. The experiments were then repeated using the fast-growing marine Gram negative species Vibrio natriegenes ${ }^{61}$. The p15a plasmid copy number is much lower $(3.0 \pm 0.5)$, presumably due to the fast growth rate and the plasmid loss is 8 -fold higher (Fig. 3e). The mRNA distribution is slightly lower, but the mean YFP is 4 -fold lower. Surprisingly, when the promoter activity is calculated $\left\langle\bar{J}_{R N A P}\right\rangle=0.015 \mathrm{RNAP} / \mathrm{s}-\mathrm{DNA}$, the distribution is nearly identical as the $E$. coli strains. Note that if this promoter were only characterized using the fluorescent output (Fig. 3h), it would have been improperly assigned a lower activity. It is surprising that this reference promoter has similar activities across these strains.

\section{Discussion}

In a single experiment, our approach is able to visualize the number of plasmids, mRNA, and protein expression levels simultaneously in living cells. Using these data, we can measure the promoter activity across a population of cells. There are several caveats including that plasmid and reporter expression that can influence the system, the assumption that mRNA degradation across cells is constant, and the pseudosteady-state approximation that mRNA/protein levels instantly adjust to changes in plasmid copy number and promoter activity.

This reference promoter can be used to convert measurements of other genetic parts (fluorescence or RNA-seq) to absolute units, facilitating their use in biophysical models of system performance ${ }^{62}$. Further, assigning a value to a promoter activity that would otherwise be reported in AU or RPU provides insight into the cellular resources needed to run a system ${ }^{63,64}$. For example, we can now estimate the RNAP usage of a 4-input genetic circuit used to make an E. coli digital display (the circuit referred to as "Segment A") requires a continuous input of 90 RNAP to maintain the lowest off state and 130 RNAP to maintain the highest on state (Supplementary Figure 17) ${ }^{13,65}$. This draws resources from a finite cellular pool of $\sim 5000$ RNAPs $^{13,59}$. Having the underlying distribution of promoter activities, rather than just the average, can be used to calculate the failure probability of a system, whether it be for a subset of cells to perform the wrong computation or accumulate a toxic intermediate due to a mismatch in enzyme levels.

\section{Methods}

Strains and media. E. coli strain NEB 10-beta [ $\triangle$ (ara-leu) 7697 araD139 fhuA $\triangle$ lacX74 galK16 galE15 e14- $\varphi 80$ dlacZDM15 recA1 relA1 endA1 nupG rpsL (Str ${ }^{R}$ ) rph spoT1 $\Delta(m r r-h s d R M S-m c r B C)$ ] was used for all cloning and experiments (New England Biolabs, C3019). Note that this strain is deficient for non-specific nuclease and recombinase. E. coli strain NEB 10-beta, E. coli MG1655 (NCBI U00096.3) and $V$. natriegens (ATCC 14048) were used for DNA/mRNA quantifications. E. coli cells were grown in LB Miller broth (Miller, BD Difco, 244620); M9 medium containing M9 salt (Sigma-Aldrich, M6030) and 0.4 \% D-glucose (Fisher Chemical, D16-1), 0.2 $\%$ casamino acids (BD Bacto, 223050), $2 \mathrm{mM} \mathrm{MgSO}_{4}$ (Affymetrix, 18651), $0.1 \mathrm{mM}$ $\mathrm{CaCl}_{2}$ (Sigma-Aldrich, C1016) and $0.34 \mathrm{~g} / \mathrm{L}$ thiamine hydrochloride (Sigma-Aldrich, T4625); or $2 \times Y$ T medium (BD, 244020). V. natriegens cells were grown in LB and M9 media with $2 \% \mathrm{NaCl}$ (VWR, 7647-14-5). $50 \mu \mathrm{g} / \mathrm{ml}$ kanamycin (Gold Biotechnology, K-120), $100 \mu \mathrm{g} / \mathrm{ml}$ ampicillin (Gold Biotechnology, MO, A-301-5) and $25 \mu \mathrm{g} / \mathrm{ml}$ chloramphenicol (VWR, AAB20841-14) were used to maintain plasmids in E. coli. $100 \mu \mathrm{g} / \mathrm{ml}$ kanamycin and $10 \mu \mathrm{g} / \mathrm{ml}$ ampicillin was used to maintain plasmid in $V$. natriegens. Isopropyl $\beta$-D-1-thiogalactopyranoside (IPTG; Sigma-Aldrich, I6758), anhydrotetracycline hydrochloride (aTc; Sigma-Aldrich, 37919), vanillic acid (Van; Sigma-Aldrich, 94770) and 3-oxohexanoyl-homoserine lactone (3OC6-AHL; Sigma-Aldrich, K3007) were used to induce gene expression. $500 \mu \mathrm{g} / \mathrm{ml} \mathrm{rifampicin}$ (Sigma-Aldrich, R3501) was used to inhibit RNA synthesis. 20XPP7 binding site repeats were derived from pCR4-24XPP7SL (a gift from Robert Singer, Addgene plasmid \#31864) $)^{37}$. Hok/sok toxin-antitoxin pair was PCRed from pSC03 ${ }^{50}$ (gift from Tal Danino).

Cell growth. E. coli NEB 10-beta cells containing the plasmids of interest were streaked on LB plates (1.5\% Agar; BD, Franklin Lakes, NJ) and grown overnight at 
$37^{\circ} \mathrm{C}$. Single colonies were inoculated into $150 \mu \mathrm{LB}$ in V-bottom 96-well plate (Nunc, 249952) with antibiotics. The plates were sealed with AeraSeal film (Excel Scientific, B-100) and incubated at 1000 r.p.m. and $37^{\circ} \mathrm{C}$ in an ELMI shaker (ELMI, DTS-4) overnight. Then the overnight cultures were diluted 178-fold into $200 \mu \mathrm{l} \mathrm{M} 9$ medium in a V-bottom 96-well plate and grown at $37^{\circ} \mathrm{C}$ at 1000 r.p.m. in an ELMI shaker for three hours. Then the cells were diluted 667 -fold by adding $15 \mu \mathrm{l}$ of culture to $185 \mu \mathrm{l} \mathrm{M} 9$ media, and then $20 \mu \mathrm{l}$ of that dilution to $980 \mu \mathrm{l} \mathrm{M} 9$ medium with antibiotics and inducers in deep 96-well plate (USA Scientific, 18962000) and grown at $37^{\circ} \mathrm{C}$ at 900 r.p.m. in an INFORS-HT shaker (INFORS-HT, Multitron Pro) for $5 \mathrm{~h}\left(\mathrm{OD}_{600} \sim 0.1\right)$ before performing microscopy. To quantify the transcript and plasmid copy number in stationary phase, the overnight culture was diluted 200-fold into $1 \mathrm{ml}$ growth media with appropriate antibiotics in a deep 96-well plate. Inducers were added to the cell culture after $4 \mathrm{~h}$ growth at $37^{\circ} \mathrm{C}$. Then the cells were grown for another $5 \mathrm{~h}$ to reach stationary growth phase. To quantify the transcript and plasmid copy number in $2 \times \mathrm{YT}$ media, the overnight culture was diluted 178 -fold into $200 \mu \mathrm{l} 2 \times \mathrm{YT}$ medium in a V-bottom 96-well plate and grown at $37^{\circ} \mathrm{C}$ for $3 \mathrm{~h}$. The cell cultures were diluted 5,336-fold into $1 \mathrm{ml}$ $2 \times$ YT media in deep 96 -well plate and grown for $5 \mathrm{~h}$ before microscope experiment. The same protocol was used to grow E. coli MG1655. For V. natriegens, the cells were grown overnight in $\mathrm{LB}$ media with $2 \% \mathrm{NaCl}$. Then, the cell cultures were diluted 100 -fold into $200 \mu \mathrm{l} \mathrm{M} 9$ media with $2 \% \mathrm{NaCl}$ in a V-bottom 96-well plate and grown at $37^{\circ} \mathrm{C}$ for $1 \mathrm{~h}$. Finally, the cell cultures were diluted 400 -fold into $1 \mathrm{ml}$ $\mathrm{M} 9$ medium with $2 \% \mathrm{NaCl}$ in deep 96 -well plate and grown for $3 \mathrm{~h}$ before performing microscopy.

Microscopy assay. Agarose pads were prepared using M9 medium with $1 \%$ agarose (SeaKem, 50004) ${ }^{66}$. The agarose pad was cooled to room temperature before sample preparation. To concentrate cells, $1 \mathrm{ml}$ cell culture was centrifuged at $6000 \mathrm{rcf}$ for $2 \mathrm{~min}$ in an Eppendorf microcentrifuge (Eppendorf, 5424). The supernatant was removed and the cell pellet was resuspended in $10 \mu \mathrm{l}$ M9 medium. A $1.5 \mu \mathrm{l}$ aliquot was pipetted onto a $22 \times 50 \mathrm{~mm}$ cover glass (VWR, 48393-059) and covered by an agarose pad to press the cells onto the imaging surface. Another $22 \times 22 \mathrm{~mm}$ cover glass (VWR, 48366-067) was placed on top of the agarose pads to reduce vaporization. The cell sample was put on ice when transporting from cell culture to imaging. Microcopy experiments were performed with an inverted epifluorescence microscope (Nikon Ti-E) equipped with an oil-immersion phasecontrast $100 \times$ objective (1.3 NA, CFI Plan Apochromat, Ph3). Images were taken using a fluorescence microscope camera (Andor, DR-328G-CO2-SIL). Nikon Elements software version 4.0 is used to control the microscope and export the images. Four channels are collected, from longest wavelength to shortest wavelength, to minimize crosstalk between different color channels. For the new measurement standard, the signal from RFP was imaged using a 570/40 nm excitation filter, $600 \mathrm{~nm}$ beam splitter and $645 / 75 \mathrm{~nm}$ emission filter. The signal from YFP was imaged using a 500/20 nm excitation filter, a $515 \mathrm{~nm}$ beam splitter and a $535 / 30 \mathrm{~nm}$ emission filter. CFP-labeled mRNA was imaged using a 436/20 nm excitation filter, a $455 \mathrm{~nm}$ beam splitter and a $480 / 40 \mathrm{~nm}$ emission filter. The phase contrast images were acquired using a halogen lamp set to $4 \mathrm{~V}$. For plasmid calibration experiments, the signal from GFP channel was imaged using a $470 / 40 \mathrm{~nm}$ excitation filter, a $495 \mathrm{~nm}$ beam splitter and a 525/50 nm emission filter.

Microscopy Image analysis. All the images were processed using MATLAB (The Mathworks). Schnitzcells ${ }^{67}$ was used to generate cell segmentations from images of color channels that were not used for DNA and mRNA quantification. Images from different color channels were aligned by maximizing their 2-D correlations calculated by corr2 function (MATLAB), which helps to adjust the cell masks for RFP (DNA) or CFP (mRNA) channel. Spot intensities for RFP or CFP channel were quantified using a customized MATLAB script (https://github.com/VoigtLab/ Promoter_Activity_Quantification) ${ }^{66}$. A Gaussian filter with a radius of 5 pixels was applied to smoothen the fluorescence profile for each cell (MATLAB function imfilter). Local maxima corresponding to spots were identified using MATLAB function imregionalmax. The pixel values near the maxima were fitted by $2 \mathrm{D}$ Gaussian functions with a constant fluorescence background. The fitting was done using MATLAB function lsqcurvefit with default settings. The spot intensities are quantified as the integration of fitted Gaussian functions without the constant background (Figure S1). The total protein expression (fluorescence) is calculated as the sum of all the pixels in the cell. Cell volume is calculated as the cell area multiplies the cell width. The FISH signal was calculated as the sum of all the pixels in the cell.

Time-lapse measurement. Overnight cultures of strains were grown in the same manner as for the one-time microscope assay (above). The overnight culture was diluted 1:178 into $200 \mu \mathrm{l}$ M9 media in a V-bottom 96-well plate, sealed with an AeraSeal film and grown at $37^{\circ} \mathrm{C}$ at 1000 r.p.m. in an ELMI shaker for three hours. Then the cultures were diluted 1:400 into $1 \mathrm{ml} \mathrm{M} 9$ medium with antibiotics and $6 \mathrm{ng} / \mathrm{ml}$ aTc in deep 96- well plate and grown at $37^{\circ} \mathrm{C}$ at 900 r.p.m. in an INFORSHT shaker for $4 \mathrm{~h}$. The agarose pad was prepared following the protocol of Tanenbaum and co-workers ${ }^{66}$. Time-lapse experiments were performed at $37^{\circ} \mathrm{C}$ with an inverted epifluorescence microscope (Nikon Ti-E) surrounded by a temperature-controlled enclosure. The enclosure and the imaging platform were preheated before experiments. The images were taken every $10 \mathrm{~min}$ and Nikon PerfectFocus system was used to correct focal drift. Images were acquired and the individual cells between different time frames were manually tracked.

Statistical analysis. The following protocol was followed to select the cells from images for analysis. All cells are first identified in the image using Schnitzcells and then a subset of $n$ cells are selected randomly using randperm function (MATLAB) which ensures sampling without replacement. Replicate experiments are performed and the same number of cells are selected from the images obtained as part of each replicate and these are used to create a distribution. The population mean and standard deviation are obtained from this combined distribuiton. For day-to-day variation, the distribution for each replicate is built, the mean calculated and the means for each replicate are used to calculate the standard deviation. To determine whether the replicates are representative of the same underlying distribution, the Kolmogorov-Smirnov Smirnov test is performed for each pair of replicates and the $\mathrm{p}$ values are calculated using the kstest 2 function (MATLAB).

mRNA degradation assay. Overnight cultures of strains were grown in the same manner as for the microscope assay. The overnight culture was diluted 1:178 into $200 \mu \mathrm{l}$ M9 medium in a V-bottom 96-well plate, sealed with an AeraSeal film and grown at $37^{\circ} \mathrm{C}$ at 1000 r.p.m. in an ELMI shaker for three hours. Then the cultures were diluted 1:667 into $1 \mathrm{ml} \mathrm{M9}$ medium with antibiotics and $6 \mathrm{ng} / \mathrm{ml}$ aTc in deep 96- well plate and grown at $37^{\circ} \mathrm{C}$ at 900 r.p.m. in an INFORS-HT shaker. After $5 \mathrm{~h}$, rifampicin was added to the cultures to a final concentration of $500 \mu \mathrm{g} / \mathrm{ml}$. The cell cultures were kept in a $37^{\circ} \mathrm{C}$ dry bath. At different time points $(0 \mathrm{~min}, 4 \mathrm{~min}$, $10 \mathrm{~min}, 20 \mathrm{~min}, 60 \mathrm{~min}$ ), cells were fixed by adding $500 \mu \mathrm{l}$ formaldehyde stock (3.5\%) to $1 \mathrm{ml}$ cell culture. The cell cultures were vortexed (Scientific Industrial, SI0236) and placed on ice. Then the cells were washed with cold phosphate buffered saline (PBS; Omnipur, 6505-OP) three times before microscope assay.

QPCR measurement. The strains were grown in the same manner as for the microscope assay. After 5-hour growth, $20 \mu \mathrm{l}$ of cell culture was boiled at $95^{\circ} \mathrm{C}$ for $5 \mathrm{~min} .1 \mu \mathrm{l}$ cell lysate was used in a $20 \mu \mathrm{l}$ reaction system using FastStart Essentia DNA Green Master (Roche, 0640271200). Primers that amplify a region of Amp resistance gene and $d x s$ gene in the terminus region of genome was used to quantify copy number of plasmid relative to the copy number of terminus region, which is assumed to be 1 copy per cell ${ }^{48}$. The qPCR experiment was run in a LightCycler 96 with SW 1.1 (Roche) and the plasmid copy number was calculated using the $\Delta \Delta \mathrm{Ct}$, assuming an efficiency of $100 \%$.

Flow cytometry assay. The cell culture was diluted $1: 10$ by adding $20 \mu \mathrm{l}$ of cell culture into $180 \mu \mathrm{l}$ of PBS containing $2 \mathrm{mg} / \mathrm{ml} \mathrm{Kan}$. Fluorescence was measured using the LSRII Fortessa flow cytometer (BD Biosciences). The experiment was run in standard mode at a flow rate of $0.5 \mu \mathrm{l} / \mathrm{s}$. The FlowJo software version 7.6 (TreeStar) was used to gate the events using forward and side scatter (Supplementary Fig. 16). For each sample, at least 50,000 of cells were used for analysis and the median fluorescence value was recorded.

FISH sample preparation. Six 20 base-pair oligonucleotide probes that target the PP7 binding sequence were designed using Stellaris Probe Designer version 4.1 (https://www.biosearchtech.com/products/rna-fish/custom-stellaris-probe-sets) and ordered from Biosearch Technologies (Hoddesdon, UK). E. coli NEB 10-beta cells containing the plasmids of interest were streaked on LB plates and grown overnight at $37^{\circ} \mathrm{C}$. Single colonies were inoculated into $4 \mathrm{ml}$ of $2 \times \mathrm{YT}$ medium in $15 \mathrm{ml}$ culture tubes (Falcon, 352059). The cells were grown overnight for $16 \mathrm{~h}$ at 37 ${ }^{\circ} \mathrm{C}$ in an incubator shaking at 300 r.p.m. (Benchmark Scientific, Incu-shaker Mini). Then, the cultures were diluted 1:300 into $25 \mathrm{ml} \mathrm{M9}$ media with antibiotics and inducers in a $50 \mathrm{ml}$ conical centrifuge tube (Falcon, 352070) with a screw-top lid $1 / 4$ closed. The cultures were incubated at $37^{\circ} \mathrm{C}$ for $4 \mathrm{~h}$ shaking at 300 r.p.m. (Benchmark Scientific, Incu-shaker Mini), after which $4 \mathrm{ml}$ of each culture was aliquoted into each of 5 different $15 \mathrm{ml}$ culture tubes kept in a $37^{\circ} \mathrm{C}$ dry bath in a chemical fume hood for measuring 5 different time points. $20 \mu \mathrm{lof} 100 \mathrm{mg} / \mathrm{ml}$ rifampicin was added to every $4 \mathrm{ml}$ of cell culture and vortexed for $3 \mathrm{~s}$ to mix (Scientific Industries, Vortex Genie). At different time points $(0,2,4,20$, and $120 \mathrm{~min}$ ), cells were fixed by adding $2 \mathrm{ml}$ formaldehyde (3.7\% by weight, diluted from stock 1:10 into ice cold $1 \times \mathrm{PBS}$ ) to $4 \mathrm{ml}$ cell culture and pipette-mixed. The culture tube was immediately put on ice. After cultures for all time points were fixed and on ice, cells were washed twice with $1 \times \mathrm{PBS}$, resuspended in $85 \%$ methanol for permeabilization for $1 \mathrm{~h}$ at room temperature, and then stored at $4{ }^{\circ} \mathrm{C}$ for 2 days (at the permeabilization stage, cells can be stored at $4{ }^{\circ} \mathrm{C}$ for up to 1 week). Cells were transferred to new Eppendorf tubes, washed in a solution of $50 \%$ formamide Wash Buffer A (Biosearch Technologies, SMF-WA1-60), and then resuspended in $40 \mu \mathrm{l}$ of $50 \%$ formamide Hybridization Buffer (Biosearch Technologies, SMF-HB1-10) containing 1.25 $\mu$ M PP7 FISH probe. Hybridized samples were incubated at $30^{\circ} \mathrm{C}$ for $16 \mathrm{~h}$, and then stored at $4{ }^{\circ} \mathrm{C}$ for 6 weeks. Cells were washed 3 times with $50 \%$ formamide Wash Buffer A, resuspended in $100 \mu$ l DAPI at $10 \mu \mathrm{g} / \mathrm{ml}$, and incubated at $30^{\circ} \mathrm{C}$ for $30 \mathrm{~min}$ to label DNA. Cells were then washed in $500 \mu \mathrm{l}$ Wash Buffer B (Biosearch Technologies, SMF-WB1-20), and resuspended in $5 \mu \mathrm{l}$ freshly-filtered $2 \times$ SSC buffer (Ambion, AM9763) for imaging. 
FISH assay. For each sample, $2 \mu$ of cells was pipetted onto a \#1 coverslip (45 mm $\times 50 \mathrm{~mm}$, Fisher Scientific, \#12-544 F). A 1.5\% agarose pad was placed on top of the sample droplet to press the cells onto the imaging surface, and another, smaller, \#1 coverslip $(22 \mathrm{~mm} \times 22 \mathrm{~mm}$, Fisher Scientific, \#12-545B) was placed on top of the agarose pad. Imaging was performed using an inverted epifluorescence microscope (Zeiss Axio Observer.Z1) with a $100 \times 1.46$ NA oil-immersion phase-contrast objective lens (Zeiss, alpha Plan-Apochromat Ph3 M27) and a cooled digital CMOS camera (Hamamatsu Orca Flash 4.0). Zen Pro software was used to control microscope and camera. Five channels were collected in the following sequence, from longest to shortest wavelength to minimize effects of cross-talk. In Channel 1, TAMRA fluorescence was collected using excitation from an HXP $120 \mathrm{~W}$ mercury arc lamp at $100 \%$ intensity, with a $550 \pm 12 \mathrm{~nm}$ excitation filter, a $570 \mathrm{~nm}$ beamsplitter, and a $605 \pm 35 \mathrm{~nm}$ emission filter. For this channel, $9 \mathrm{z}$-slices were collected at a spacing of $200 \mathrm{~nm}$ per slice (total $\mathrm{z}$-range of $1.6 \mu \mathrm{m}$ ), with an integration time of $1 \mathrm{~s}$ per slice. In Channel 2, YFP fluorescence was collected using LED excitation at $470 \mathrm{~nm}$ (Zeiss Colibri, $100 \%$ intensity), with a $470 \pm 20 \mathrm{~nm}$ excitation filter, a $495 \mathrm{~nm}$ beamsplitter, and a $525 \pm 25$ emission filter, at a single $\mathrm{z}$ slice with an integration time of $1 \mathrm{~s}$. In Channel 3, CFP fluorescence was collected using excitation from an HXP $120 \mathrm{~W}$ mercury arc lamp at $100 \%$ intensity, with a $436 \pm 12 \mathrm{~nm}$ excitation filter, a $455 \mathrm{~nm}$ beamsplitter, and a $480 \pm 20 \mathrm{~nm}$ emission filter, at a single z-slice with an integration time of $1 \mathrm{~s}$. In Channel 4 , DAPI fluorescence was collected using LED excitation at $385 \mathrm{~nm}$ (Zeiss Colibri, 25\% intensity), with a $359 \pm 24 \mathrm{~nm}$ excitation filter, a $395 \mathrm{~nm}$ beamsplitter, and a $445 \pm$ 25 emission filter, at a single z-slice with an integration time of $50 \mathrm{~ms}$. In Channel 5 , phase contrast was used to image bacterial cell bodies using a halogen lamp set to $4 \mathrm{~V}$, collected over $9 \mathrm{~s}$-slices separated by $200 \mathrm{~nm}$ each (total z-range of $1.6 \mu \mathrm{m}$ ), with an integration time of $100 \mathrm{~ms}$ per slice. Each sample was imaged at a minimum of 3 different locations. Images were exported as TIFF files for subsequent analysis.

Measurement of cell growth. Overnight cultures of strains were grown in the same manner as for the microscope assay. Briefly the overnight culture was diluted 1:178 into $200 \mu \mathrm{l} \mathrm{M} 9$ medium in a V-bottom 96-well plate, and grown at $37^{\circ} \mathrm{C}$ at 1000 r.p.m. in an ELMI shaker for three hours. Then the cultures were diluted 1:667 into $1 \mathrm{ml} \mathrm{M9}$ medium with antibiotics and $6 \mathrm{ng} / \mathrm{ml}$ aTc in a deep 96- well plate and grown at $37^{\circ} \mathrm{C}$ in an INFORS-HT shaker. Starting from $4 \mathrm{~h}$ after incubation, the $\mathrm{OD}_{600}$ of the sample was measured every $20 \mathrm{~min}$ in a plate reader (Synergy H1 microplate reader, Biotek) for $2 \mathrm{~h}$. The doubling time was calculated by assuming exponential growth in this time period.

Reporting summary. Further information on research design is available in the Nature Research Reporting Summary linked to this article.

\section{Data availability}

Source data are provided with this paper. Genetic part sequences are available in Supplementary Information. Plasmids are available from Addgene. Any other relevant data are available from the corresponding author upon reasonable request.

\section{Code availability}

Matlab scripts used for image processing are released as open - source software under the MIT license (GitHub repository: https://github.com/VoigtLab/

Promoter_Activity_Quantification).

Received: 26 June 2020; Accepted: 8 February 2021;

Published online: 05 March 2021

\section{References}

1. Endy, D. Foundations for engineering biology. Nature 438, 449-453 (2005)

2. Goldman, S. R., Ebright, R. H. \& Nickels, B. E. Direct detection of abortive RNA transcripts in vivo. Science 324, 927-928 (2009).

3. Li, G.-W. \& Xie, X. S. Central dogma at the single-molecule level in living cells. Nature 475, 308-315 (2011).

4. So, L.-h et al. General properties of transcriptional time series in Escherichia coli. Nat. Genet. 43, 554-560 (2011).

5. Khalil, A. S. \& Collins, J. J. Synthetic biology: applications come of age. Nat. Rev. Genet. 11, 367-379 (2010).

6. Purnick, P. E. M. \& Weiss, R. The second wave of synthetic biology: from modules to systems. Nat. Rev. Mol. Cell Bio. 10, 410-422 (2009).

7. Nielsen, A. A. et al. Genetic circuit design automation. Science 352, aac7341 (2016).

8. Jeschek, M., Gerngross, D. \& Panke, S. Combinatorial pathway optimization for streamlined metabolic engineering. Curr. Opin. Biotechnol. 47, 142-151 (2017).
9. Beal, J. et al. TASBE flow analytics: a package for calibrated flow cytometry analysis. ACS Synth. Biol. 8, 1524-1529 (2019).

10. Kelly, J. R. et al. Measuring the activity of BioBrick promoters using an in vivo reference standard. J. Biol. Eng. 3, 4 (2009).

11. Iyer, S., Park, B. R. \& Kim, M. Absolute quantitative measurement of transcriptional kinetic parameters in vivo. Nucleic Acids Res. 44, e142 (2016)

12. Friedman, LarryJ. \& Gelles, J. Mechanism of transcription initiation at an activator-dependent promoter defined by single-molecule observation. Cell 148, 679-689 (2012).

13. Schafer, D. A., Gelles, J., Sheetz, M. P. \& Landick, R. Transcription by single molecules of RNA polymerase observed by light microscopy. Nature 352, 444-448 (1991).

14. Wang, H. et al. CRISPR-mediated live imaging of genome editing and transcription. Science 365, 1301-1305 (2019).

15. Elowitz, M. B., Levine, A. J., Siggia, E. D. \& Swain, P. S. Stochastic gene expression in a single cell. Science 297, 1183-1186 (2002).

16. Paulsson, J. Summing up the noise in gene networks. Nature 427, 415-418 (2004).

17. Albayrak, C. et al. Digital quantification of proteins and mRNA in single mammalian cells. Mol. Cell 61, 914-924 (2016)

18. Wang, Y., Penkul, P. \& Milstein, J. N. Quantitative localization microscopy reveals a novel organization of a high-copy number plasmid. Biophys. J. 111, 467-479 (2016).

19. Gorochowski, T. E. et al. Genetic circuit characterization and debugging using RNA-seq. Mol. Syst. Biol. 13, 952 (2017).

20. Wang, J., Chen, L., Chen, Z. \& Zhang, W. RNA-seq based transcriptomic analysis of single bacterial cells. Integr. Biol. 7, 1466-1476 (2015).

21. Kang, Y. et al. Transcript amplification from single bacterium for transcriptome analysis. Genome Res. 21, 925-935 (2011).

22. Wegrzyn, G. Replication of plasmids during bacterial response to amino acid starvation. Plasmid 41, 1-16 (1999).

23. Lin-Chao, S. \& Bremer, H. Effect of the bacterial growth rate on replication control of plasmid pBR322 in Escherichia coli. Mol. Gen. Genet 203, 143-149 (1986)

24. Park, Y., Espah Borujeni, A., Gorochowski, T. E., Shin, J. \& Voigt, C. A. Precision design of stable genetic circuits carried in highly-insulated E. coli genomic landing pads. Mol. Syst. Biol. 16, e9584 (2020).

25. Chandler, M. G. \& Pritchard, R. H. The effect of gene concentration and relative gene dosage on gene output in Escherichia coli. Mol. Gen. Genet 138 127-141 (1975)

26. Tal, S. \& Paulsson, J. Evaluating quantitative methods for measuring plasmid copy numbers in single cells. Plasmid 67, 167-173 (2012).

27. Wang, Y. Spatial distribution of high copy number plasmids in bacteria. Plasmid 91, 2-8 (2017).

28. Yao, S., Helinski, D. R. \& Toukdarian, A. Localization of the naturally occurring plasmid ColE1 at the cell pole. J. Bacteriol. 189, 1946-1953 (2007)

29. Pogliano, J., Ho, T. Q., Zhong, Z. \& Helinski, D. R. Multicopy plasmids are clustered and localized in Escherichia coli. Proc. Natl Acad. Sci. USA 98, 4486-4491 (2001)

30. Gaal, T. et al. Colocalization of distant chromosomal loci in space in E. coli: a bacterial nucleolus. Genes Dev. 30, 2272-2285 (2016).

31. Reyes-Lamothe, R. et al. High-copy bacterial plasmids diffuse in the nucleoidfree space, replicate stochastically and are randomly partitioned at cell division. Nucleic Acids Res. 42, 1042-1051 (2013).

32. Gordon, G. S. et al. Chromosome and low copy plasmid segregation in E. coli: visual evidence for distinct mechanisms. Cell 90, 1113-1121 (1997).

33. Diaz, R., Rech, J. \& Bouet, J.-Y. Imaging centromere-based incompatibilities: Insights into the mechanism of incompatibility mediated by low-copy number plasmids. Plasmid 80, 54-62 (2015).

34. Jahn, M., Vorpahl, C., Hübschmann, T., Harms, H. \& Müller, S. Copy number variability of expression plasmids determined by cell sorting and Droplet Digital PCR. Microb. Cell Factories 15, 211 (2016).

35. Friehs, K. in New Trends and Developments in Biochemical Engineering 47-82 (Springer, 2004)

36. Bertrand, E. et al. Localization of ASH1 mRNA particles in living yeast. Mol Cell 2, 437-445 (1998).

37. Larson, D. R., Zenklusen, D., Wu, B., Chao, J. A. \& Singer, R. H. Real-time observation of transcription initiation and elongation on an endogenous yeast gene. Science 332, 475-478 (2011)

38. Yan, X., Hoek, T. A., Vale, R. D. \& Tanenbaum, M. E. Dynamics of translation of single mRNA molecules in vivo. Cell 165, 976-989 (2016).

39. Tutucci, E. et al. An improved MS2 system for accurate reporting of the mRNA life cycle. Nat. Methods, https://doi.org/10.1038/nmeth.4502 (2017).

40. Golding, I., Paulsson, J., Zawilski, S. M. \& Cox, E. C. Real-time kinetics of gene activity in individual bacteria. Cell 123, 1025-1036 (2005).

41. Kandhavelu, M., Häkkinen, A., Yli-Harja, O. \& Ribeiro, A. S. Single-molecule dynamics of transcription of the lar promoter. Phys. Biol. 9, 026004 (2012).

42. Khuperkar, D. et al. Quantification of mRNA translation in live cells using single-molecule imaging. Nat. Protoc., https://doi.org/10.1038/s41596-0190284-x (2020). 
43. Chao, J. A., Patskovsky, Y., Almo, S. C. \& Singer, R. H. Structural basis for the coevolution of a viral RNA-protein complex. Nat. Struct. Mol. Biol. 15, 103-105 (2008).

44. Janicki, S. M. et al. From silencing to gene expression: real-time analysis in single. Cells Cell 116, 683-698 (2004).

45. Hocine, S., Raymond, P., Zenklusen, D., Chao, J. A. \& Singer, R. H. Singlemolecule analysis of gene expression using two-color RNA labeling in live yeast. Nat. Meth 10, 119-121 (2013).

46. Koch, A., Aguilera, L., Morisaki, T., Munsky, B. \& Stasevich, T. J. Quantifying the spatiotemporal dynamics of IRES versus Cap translation with single-molecule resolution in living cells. https://doi.org/10.1101/2020.01.09.900829 (2020).

47. Abbas, A. et al. Characterization of interactions between the transcriptional repressor $\mathrm{PhlF}$ and its binding site at the $<\mathrm{em}>\mathrm{phlA}</ \mathrm{em}>$ Promoter in $<\mathrm{em}>$ Pseudomonas fluorescens $</ \mathrm{em}>$ F113. J. Bacteriol. 184, 3008-3016 (2002).

48. Lee, C., Kim, J., Shin, S. G. \& Hwang, S. Absolute and relative QPCR quantification of plasmid copy number in Escherichia coli. J. Biotechnol. 123, 273-280 (2006).

49. Summers, D. K. \& Sherratt, D. J. Multimerization of high copy number plasmids causes instability: CoIE1 encodes a determinant essential for plasmid monomerization and stability. Cell 36, 1097-1103 (1984).

50. Chowdhury, S. et al. Programmable bacteria induce durable tumor regression and systemic antitumor immunity. Nat. Med. 25, 1057-1063 (2019).

51. Paulsson, J. \& Ehrenberg, M. Noise in a minimal regulatory network: plasmid copy number control. Q Rev. Biophys. 34, 1-59 (2001).

52. Goss, P. J. E. \& Peccoud, J. in Biocomputing '99 65-76 (WORLD SCIENTIFIC, 1998).

53. Paulsson, J. \& Ehrenberg, M. Trade-off between segregational stability and metabolic burden: a mathematical model of plasmid ColE1 replication control11Edited by D Draper. J. Mol. Biol. 279, 73-88 (1998).

54. Jones, D. L., Brewster, R. C. \& Phillips, R. Promoter architecture dictates cellto-cell variability in gene expression. Science 346, 1533-1536 (2014).

55. Wang, M., Zhang, J., Xu, H. \& Golding, I. Measuring transcription at a single gene copy reveals hidden drivers of bacterial individuality. Nat. Microbiol., https://doi.org/10.1038/s41564-019-0553-z (2019).

56. Chong, S., Chen, C., Ge, H. \& Xie, X. S. Mechanism of transcriptional bursting in bacteria. Cell 158, 314-326 (2014).

57. Yarchuk, O., Jacques, N., Guillerez, J. \& Dreyfus, M. Interdependence of translation, transcription and mRNA degradation in the lacZ gene. J. Mol. Biol. 226, 581-596 (1992).

58. Peccoud, J. \& Ycart, B. Markovian modeling of gene-product synthesis. Theor. Popul. Biol. 48, 222-234 (1995).

59. Grigorova, I. L., Phleger, N. J., Mutalik, V. K. \& Gross, C. A. Insights into transcriptional regulation and sigma competition from an equilibrium model of RNA polymerase binding to DNA. Proc. Natl Acad. Sci. USA 103, 5332-5337 (2006).

60. Churchward, G., Bremer, H. \& Young, R. Transcription in bacteria at different DNA concentrations. J. Bacteriol. 150, 572-581 (1982).

61. Weinstock, M. T., Hesek, E. D., Wilson, C. M. \& Gibson, D. G. Vibrio natriegens as a fast-growing host for molecular biology. Nat. Meth 13, 849-851, http://www.nature.com/nmeth/journal/v13/n10/abs/nmeth.3970. html\#supplementary-information (2016).

62. Espah Borujeni, A., Zhang, J., Doosthosseini, H., Nielsen, A. A. K. \& Voigt, C. A. Genetic circuit characterization by inferring RNA polymerase movement and ribosome usage. Nat. Commun. 11, 5001 (2020).

63. Gorochowski, T. E. et al. Absolute quantification of translational regulation and burden using combined sequencing approaches. Mol. Syst. Biol. 15, e8719 (2019).

64. Ceroni, F., Algar, R., Stan, G. B. \& Ellis, T. Quantifying cellular capacity identifies gene expression designs with reduced burden. Nat. Methods 12 415-418 (2015).
65. Kim, S., Beltran, B., Irnov, I. \& Jacobs-Wagner, C. Long-distance cooperative and antagonistic RNA polymerase dynamics via DNA supercoiling. Cell 179, 106-119 (2019). e116.

66. Skinner, S. O., Sepulveda, L. A., Xu, H. \& Golding, I. Measuring mRNA copy number in individual Escherichia coli cells using single-molecule fluorescent in situ hybridization. Nat. Protoc. 8, 1100-1113 (2013).

67. Young, J. W. et al. Measuring single-cell gene expression dynamics in bacteria using fluorescence time-lapse microscopy. Nat. Protoc. 7, 80-88 (2011).

\section{Acknowledgements}

This work was supported by US National Institutes of Standards and Technology grant no. 70-NANB16H164 (B.S., J.R. and C.A.V.), US Department of Energy grant no. DE-FOA-0001650 (B.S. and C.A.V.), and the National Research Council Postdoctora Associateship (J.R.). The National Institute of Standards and Technology notes that certain commercial equipment, instruments, and materials are identified in this paper to specify an experimental procedure as completely as possible. In no case does the identification of particular equipment or materials imply a recommendation or endorsement by NIST, nor does it imply that the materials, instruments, or equipment are necessarily the best available for the purpose.

\section{Author contributions}

C.A.V., B.S. and D.R. conceived the study, designed the experiments and wrote the manuscript. B.S. and D.A.A. constructed the plasmids and carried out microscope experiments. J.R., B.S. and N.A. performed FISH experiments.

\section{Competing interests}

The authors declare no conflict of interests.

\section{Additional information}

Supplementary information The online version contains supplementary material available at https://doi.org/10.1038/s41467-021-21734-y.

Correspondence and requests for materials should be addressed to C.A.V.

Peer review information Nature Communications thanks Brian Munksy and the other, anonymous, reviewer(s) for their contribution to the peer review of this work. Peer reviewer reports are available.

Reprints and permission information is available at http://www.nature.com/reprints

Publisher's note Springer Nature remains neutral with regard to jurisdictional claims in published maps and institutional affiliations.

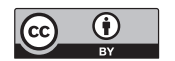

Open Access This article is licensed under a Creative Commons Attribution 4.0 International License, which permits use, sharing, adaptation, distribution and reproduction in any medium or format, as long as you give appropriate credit to the original author(s) and the source, provide a link to the Creative Commons license, and indicate if changes were made. The images or other third party material in this article are included in the article's Creative Commons license, unless indicated otherwise in a credit line to the material. If material is not included in the article's Creative Commons license and your intended use is not permitted by statutory regulation or exceeds the permitted use, you will need to obtain permission directly from the copyright holder. To view a copy of this license, visit http://creativecommons.org/ licenses/by/4.0/.

(c) The Author(s) 2021 